\title{
Excellent femoral outcomes when all access attempts and closure devices are performed by experienced cardiologists
}

\author{
Kevin S. Kang, Quentin Orlando, Robert Maholic, Richard Petrella, Gurjaipal S. Kang* \\ UPMC Hamot Hospital, Erie, USA \\ Email: *drpaulkang@gmail.com
}

Received 25 September 2013; revised 27 October 2013; accepted 16 November 2013

Copyright (c) 2013 Kevin S. Kang et al. This is an open access article distributed under the Creative Commons Attribution License, which permits unrestricted use, distribution, and reproduction in any medium, provided the original work is properly cited.

\begin{abstract}
Femoral access is considered less safe for access site complications than the radial access. Cardiovascular procedures have not been studied taking operator experience, defined as American Board of Internal Medicine, Interventional Cardiology certification or equivalent qualification in another country, into account. We hypothesize that the procedural results are operator dependent and excellent results are obtained when procedures are performed by experienced operators. Femoral access is higher risk than radial access based on American College of Cardiology (ACC) guidelines. Femoral access is less forgiving, as opposed to radial, as it is an end-artery, lacks easy compressibility and is more likely to cause morbidity when injured. Hence, radial is recommended over the femoral approach according to ACC practice guidelines. These guidelines are often based on the randomized studies from academic centers where trainees, with variable arterial access experience, perform the initial access stick and arterial closure device deployment. Methods: We performed a single center retrospective review of 32,446 consecutive patients undergoing invasive cardiovascular procedures done from the femoral approach using American College of Cardiology/National Cardiovascular Data Registry (ACC/NCDR) from January 1, 2006 to June 30, 2013. Only experienced operators performed the actual access site stick and the reminder of the invasive procedure. Results: Total bleeding and vascular complications were less than $1 \%$. We define outcomes as excellent if the total bleeding and vascular complication risk is less than $1 \%$ based on previous studies discussed in the ACC guidelines. Conclusion: Excellent outcomes can be obtained from the femoral access if experienced cardiologists perform the procedure. Hence, radial arterial access over the femoral access

\footnotetext{
${ }^{*}$ Corresponding author.
}

may be selectively rather than universally recommended considering the possibility of varying level of femoral access expertise of different practices.

Keywords: Operators; Access; Complications

\section{INTRODUCTION}

Cardiac diagnostic and interventional procedures have historically been performed using femoral arterial access [1]. Associated risks include access site bleeding and major vascular complications [2]. Radial access is favored to decrease the vascular complication risk during these cardiovascular procedures [3,4]. However, there are also some limitations pertaining to the radial artery. It is associated with an increased radiation exposure to the operator [5] and a higher risk of procedural failure due to the smaller size and tortuous nature of the vessel [6]. It may be harder to palpate the vessel in hypotensive patients and impossible to use with large diameter devices. The American College of Cardiology (ACC) guidelines increasingly recommend the use of the radial approach [4]. The ACC guidelines are based on studies done exclusively at academic centers where often a trainee, instead of a board certified interventional cardiologist, sticks the access artery and performs the closure device procedure. These studies comparing femoral and radial accesses fail to take the operator experience into account when reporting outcomes [3]. We defined an experienced operator as a board certified interventional cardiologist because of three reasons. Firstly, a board certified cardiologist on average is more likely to have performed greater number of procedures compared to a trainee. Secondly, the board certified attending cardiologist assumes full responsibility of patient outcomes as opposed to trainees or other staff where the ultimate responsibility still resides with the attending cardiologists in the training program. Also, board certification assures a complete know- 
ledge base of invasive cardiovascular procedures. For the purpose of our study, we define an excellent outcome as a complication risk of less than $1 \%$. We chose a number of less than $1 \%$ total vascular complication risk as an indicator on an excellent outcome based on the prior results from radial access studies and guideline recommendations [3,4]. Coronary and carotid stenting procedures are dependent on operator volume [7,8]. We hypothesize that any invasive cardiovascular procedure is operator dependent and excellent results are possible with femoral access when conducted by experienced operators. The prior randomized studies may have favored the radial approach because the arterial access was obtained by the trainees in academic centers where such studies were conducted. This lack of experience of a trainee may be associated with arterial injury to radial or the femoral artery, leading to further complications and less than excellent outcomes. We define arterial injury as causing arterial dissection, perforation or emboli during the course of access or closure device placement. However, such vascular injuries in the case of radial artery may be asymptomatic. This is because the radial artery access may be a forgiving approach to cardiac interventional and diagnostic procedures because it is superficial, easily compressible and is not an end artery [9]. If a trainee with minimal experience in arterial access injures the radial artery, the clinical outcomes are not likely to be affected. However, a femoral arterial injury is more likely to result in symptomatic post procedure sequel, such as severe bleeding or ischemic complications. Femoral artery is an end artery and is not easily compressible for hemostasis. Prior studies comparing the two access sites have favored radial over femoral without accounting for operator experience [3]. If an injury, such as dissection, of the femoral artery is avoided, the vascular outcomes with femoral artery access should be excellent. Operator experience may be important in avoiding femoral artery injury. Femoral artery may be preferred in several settings because of its larger diameter, frequent lack of tortuosity and easier palpation in hypotensive patients [9]. When experienced operators conduct femoral access procedures, total bleeding and vascular complications may be less than $1 \%$. The training level of the operator can greatly mitigate the bleeding and groin complication risks in patients undergoing femoral approach invasive procedures [7,8].

\section{METHODS}

All patients, presenting between January 1, 2006 and June 30, 2013, that underwent an invasive cardiovascular procedure through femoral access were included in our study. The total number of consecutive patients who were accessed via the femoral artery was 32,446. A board certified interventional cardiologist does all patient arterial access in our institution and the study was exclusively done at our institution. The data reported in this study is taken from the American College of Cardiology/National Cardiovascular Data Registry (ACC/ NCDR). The NCDR Registry is an initiative of the American College of Cardiology (ACC) and the Society for Cardiovascular Angiography and Interventions and has been previously described [10]. ACC committee prospectively defined the variables, which are available at www.ncdr.com (AUGUST 2013). The ACC/ NCDR has specific definitions for endpoints such as bleeding, dissection, emboli, fistula and pseudo-aneurysm, and such definitions are available on the website and are summarized below. Bleeding Complication: Blood loss at the site of arterial or venous access, or due to perforation of a traversed artery or vein requiring transfusion and/or prolonging the hospital stay, and/or causing a drop in hemoglobin of $>3.0 \mathrm{~g} / \mathrm{dL}$. Bleeding attributable to the vascular site could be retroperitoneal (retroperitoneal bleeding), a local hematoma $>10 \mathrm{~cm}$ with femoral access, $>2 \mathrm{~cm}$ with radial access, or $>5 \mathrm{~cm}$ with brachial access (hematoma bleeding), or external (entry site bleeding). Vascular Complication: This category included the presence of any one of the following vascular complications pertaining to the percutaneous access site: occlusion, defined as total obstruction of the artery by thrombus, usually at the site of access requiring surgical repair; embolization, defined as loss of distal pulse, pain and/or discoloration (especially the toes); dissection, defined as a disruption of an arterial wall resulting in splitting and separation of the intimal (subintimal) layers; pseudoaneurysm, defined as the occurrence of a disruption and dilation of the arterial wall without identification of the arterial wall layers at the site of the catheter entry demonstrated by arteriography or ultrasound; or AV fistula, defined as a connection between the access artery and the accompanying vein demonstrated by arteriography or ultrasound and most often characterized by a continuous bruit. Bleeding or Vascular Complication: Either one or the other, or both. For the purpose of our study, we use the NCDR/ACC registry limited to data reported from our single institution. The study data is limited to our center where only the nursing staff, trained in the NCDR registry, is allowed to enter data. These nurses have no financial affiliation with the operators. Our institution is a private, non-teaching hospital and all operators who stick the arterial access site are board certified interventional cardiologists rather than cardiology fellows or other non-physician staff. The study was limited to patients undergoing invasive cardiovascular procedures through femoral arterial access over a $61 / 2$ year period. No patients were excluded from this group. Attempts are not made to directly compare this 
study with previous studies using statistical means because of differences in patient populations involved in previous studies even though this is possible.

\section{RESULTS}

Patients selected for our single-center retrospective review had inpatient follow up as required by NCDR [10]. 32,446 patients were selected from January 1, 2006 to June 30, 2013 with no exclusion criteria except for undergoing invasive cardiovascular procedure from femoral access. Greater than $50 \%$ of the femoral access patients had Perclose or Starclose Closure Device deployment in the end. Only board certified cardiologists were allowed to attempt arterial access and closure device deployment at our center. Our results reveal that there was low mortality $(<0.9 \%)$ in this large patient population undergoing femoral access by experienced operators. Vascular complications recorded were limited to bleeding (0.6\%), dissections $(0.1 \%)$, occlusions $(0.0 \%)$, pseudo-aneurysms $(0.1 \%)$, emboli $(0.0 \%)$ and fistulae $(0.0 \%)$. The overall risk of vascular complications with femoral access was less than $1 \%$ and numerically lower than the risk reported in previous studies [3]. The largest randomized study, RIVAL, that compared the two access sites reported vascular complications for radial access at $1.4 \%$ and femoral access at 3.4\% [3]. The complication risk in our study involving femoral access patients was less than $1 \%$. In our study, the patients were noted to have no femoral infections. Table 1 lists values for bleeding and vascular complications.

\section{DISCUSSION}

ACC/AHA guidelines advocate radial access over the femoral access to reduce the vascular complication risk [4]. A lot of prior data in the radial versus femoral literature has been collected from teaching hospitals where

Table 1. Complications of invasive procedures done from the femoral access.

\begin{tabular}{cc}
\hline Total invasive femoral access procedures & 32,446 \\
\hline Mortality & $0.9 \%$ \\
Bleeding & $0.6 \%$ \\
Dissection & $0.1 \%$ \\
Occlusion & $0.0 \%$ \\
Pseudoaneurysm & $0.1 \%$ \\
Emboli & $0.0 \%$ \\
Fistulae & $0.0 \%$ \\
Total Bleeding and Vascular Complications & $0.8 \%$ \\
\hline
\end{tabular}

trainees with minimal experience may attempt arterial access and closure devices under the supervision of experienced cardiologists. The trainees may perform the actual arterial access stick. The impact of trainees in getting arterial access for cardiovascular procedures has not been studied, to our knowledge, but in other specialties, such as general surgery, the effect of trainees may increase the complication risk [11]. Major studies comparing outcomes with different types of arterial access were done in teaching hospitals where trainees often get arterial access and perform part of the invasive procedure albeit under supervision [3]. The ACC/AHA guidelines are based on such studies and recommend radial access [4]. The guidelines are then applied to all types of practice patterns regardless of the training experience of the operators. We realized that major studies like RIVAL may not apply to our hospital. Our institution is unique because only board certified cardiologists perform all invasive procedure and closure devices. We decided to look exclusively at our own hospital data so we could learn more about the femoral vascular complication results in cardiology practices similar to ours where only experienced operators performed the procedures. As far as we know, similar data have not been published before. In addition to access site complications associated with operator experience, closure device complications may also be operator dependent. The previously published studies often had trainees placing closure devices possibly leading to higher risk of vascular femoral site complications. So, we expect that the overall vascular outcomes associated with femoral access may be dependent on the initial access stick and the final closure device technique. Our results as noted above confirm our excellent outcomes with femoral approach in an especially high-risk population. The excellent outcomes of $<1 \%$ complication risk in our study, as predicted, may be due to differences in the operator skill. Our operators were board certified interventional cardiologists and no trainees or other non-physician staff was allowed to stick or close the artery. Our study demonstrates that when only board certified cardiologists perform access arterial stick, the injury to the femoral artery may be decreased and as a consequence, vascular and bleeding outcomes are excellent.

\section{LIMITATIONS}

Retrospective nature of the study and based in only one center.

\section{CONCLUSION}

We conclude that if only experienced operators stick an access site and personally perform the entire procedure, excellent outcomes in patients with femoral access are 
possible. Our recommendation would not generalize the clinical trial results from training centers to every cardiology practice worldwide and endorse radial over the femoral approach but acknowledge differences between practices' expertise in a particular access site.

\section{REFERENCES}

[1] Gruentzig, A. (1978) Transluminal dilatation of coronary-artery stenosis. Lancet, 1, 263. http://dx.doi.org/10.1016/S0140-6736(78)90500-7

[2] Doyle, B.J., Rihal, C.S., Gastineau, D.A. and Holmes, D.R., Jr. (2009) Bleeding, blood transfusion, and increased mortality after percutaneous coronary intervenetion: implications for contemporary practice. Journal of the American College of Cardiology, 53, 2019-2027. http://dx.doi.org/10.1016/j.jacc.2008.12.073

[3] Jolly, S.S., Yusuf, S., Cairns, J., et al. (2011) RIVAL trial group. Radial versus femoral access for coronary angiography and intervention in patients with acute coronary syndromes (RIVAL): A randomized, parallel group, multicenter trial. Lancet, 377, 1409-1420. http://dx.doi.org/10.1016/S0140-6736(11)60404-2

[4] Levine, G.N., Bates, E.R., Blankenship, J.C., et al. (2011) ACCF/AHA/SCAI Guideline for percutaneous coronary intervention: A report of the American College of Cardiology Foundation/American Heart Association Task Force on Practice Guidelines and the Society for Cardiovascular Angiography and Interventions. Journal of the American College of Cardiology, 58, e44-e122. http://dx.doi.org/10.1016/j.jacc.2011.08.007

[5] Jolly, S.S., Cairns, J., Niemela, K., et al. (2013) Effect of radial versus femoral access on radiation dose and the importance of procedural volume: A substudy of the multicenter randomized RIVAL trial. JACC: Cardiovascular Interventions, 6, 258-266. http://dx.doi.org/10.1016/j.jcin.2012.10.016

[6] Biondi-Zoccai, G., Sciahbasi, A., Bodí, V., FernándezPortales, J., et al. (2013) Right versus left radial artery access for coronary procedures: An international collaborative systematic review and meta-analysis including 5 randomized trials and 3210 patients. International Journal of Cardiology, 166, 621-626. http://dx.doi.org/10.1016/j.ijcard.2011.11.100

[7] Gray, W.A., Rosenfield, K.A., Jaff, M.R., et al. (2011) Influence of site and operator characteristics on carotid artery stent outcomes: Analysis of the CAPTURE 2 (Carotid Acculink/Accunet Post Approval Trial to Uncover Rare Events) clinical study. Journal of the American College of Cardiology, 4, 235-246. http://dx.doi.org/10.1016/j.jcin.2010.10.009

[8] Kastrati, A., Neumann, F.J. and Schömig, A. (1998) Operator volume and outcome of patients undergoing coronary stent placement. Journal of the American College of Cardiology, 32, 970-976. http://dx.doi.org/10.1016/S0735-1097(98)00334-9

[9] Nicholas, R., Balaji, P. and Shah, B. (2011) Radial artery catheterization. American Heart Association, 124, e407e408.

[10] Rao, S.V., Ou, F.S., Wang, T.Y., et al. (2008) Trends in the prevalence and outcomes of radial and femoral approaches to percutaneous coronary intervention: A report from the National Cardio-vascular Data Registry. JACC: Cardiovascular Interventions, 1, 379-386. http://dx.doi.org/10.1016/j.jcin.2008.05.007

[11] Kiran, R.P., Ahmed Ali, U., Coffey, J.C., et al. (2012) Impact of resident participation in surgical operations on postoperative outcomes: National surgical quality improvement program. Annals of Surgery, 256, 469-475. http://dx.doi.org/10.1097/SLA.0b013e318265812a

\author{
ABBREVIATIONS \\ ACC: American College of Cardiology \\ NCDR: National Cardiovascular Data Registry
}

